\title{
CUALIFICACIÓN Y FORMACIÓN DE MAESTROS: UNA EXPERIENCIA PROYECTIVA
}

Por: Isabel Pérez Chaín y Orlando Durango Rueda.

Docentes de la Facultad de Ciencias Sociales y Educación. Universidad de Cartagena.

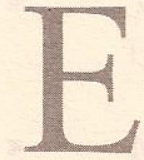

n 1995, la Facultad de Ciencias Sociales y Educación de la Universidad de Cartagena, diseñó el proyecto de Cualificación y Formación del Maestro en el Distrito de Cartagena: "Formación y Construcción de los Proyectos Educativos Institucionales", con miras a contribuir al mejoramiento de la calidad educativa de la educación básica del Distrito de Cartagena, a la luz de la ley general de educación, las demandas del contexto en formación de maestros en medio de la crisis generalizada en que vivimos y cree, como nuestro Nobel de Literatura, Gabriel García Márquez: "Que las condiciones están dadas como nunca para el cambio social y que la educación será su órgano maestro. Una educación desde la cuna hasta la tumba inconforme y reflexiva, que nos inspire un nuevo modo de pensar y nos incite a descubrir quienes somos en una sociedad que se quiera más a sí misma. Que aproveche al máximo nuestra creatividad inagotable y conciba una ética y tal vez una estética para nuestro afán desaforado y legítimo de superación personal. Que integre la ciencia y las artes a la canasta familiar, de acuerdo a los designios de un gran poeta de nuestro tiempo que pidió no seguir amándoles por separadas como a dos hermanas enemigas. Que canalice hacia la vida, la inmensa energía creativa, que durante siglos hemos despilfarrado en la depredación y la violencia y nos abra al fin la segunda oportunidad sobre la tierra que no tuvo la estirpe desgraciada del Coronel Aureliano Buendía; por el país próspero y justo que soñamos: Al alcance de los niños" ${ }^{1}$.

Conscientes del compromiso y responsabilidad social que tenemos como Facultad de Ciencias Sociales y Educación, de aportar nuestro granito de arena al proceso de cambio social a través de la educación, se concibe y origina el proyecto de Cualificación y Formación del Maestro en el Distrito de Cartagena, como una estrategia pedagógica a desarrollar en tres dimensiones:

1. García Márquez Gabriel. La Proclama: Colombia al Filo de la Oportunidad. Tercer Mundo Editores. Bogotá, 1996. P.56
- $\quad$ "Dimensión Académica, Marcos Conceptuales sobre lo Pedagógico y lo Educativo." 
- Dimensión Teórico Práctica. Brinda la Posibilidad de Articular las Concepciones educativas y pedagógicas con el trabajo participativo, de autogestión de cada Institución y su comunidad educativa.

- "Dimensión de Asesoría, seguimiento y evaluación permanente y retroalimentación de proyectos concretos, como resultado y producto de la construcción colectiva con participación de la comunidad".

Enfocado hacia la búsqueda de una educación con características como las señaladas anteriormente, en el que "se asume la educación como componente fundamental de desarrollo humano con identidad cultural y participación democrática" ${ }^{\prime 2}$

El proyecto, fue puesto a consideración de la Secretaría de Educación y Cultura Distrital de Cartagena para estudio y aprobación. En el primer semestre de 1995, siendo Secretaria de Educación y Cultura Distrital, la doctora Patricia Martínez Barrios, se aprueba y autoriza a la Facultad de Ciencias Sociales y Educación, dar inicio al proceso formativo" ${ }^{3}$, procediéndose a firmar el Convenio Interinstitucional Universidad de Cartagena-Facultad de Ciencias Sociales y Educación y el Distrito de Cartagena-Secretaría de Educación y Cultura, mediante el cual se legaliza el acto administrativo anterior.

Los docentes participantes fueron 150 de diferentes zonas, núcleos y centros educativos, quienes adquieren el compromiso con un proceso formativo, colectivo y constante cuya estructura y desarrollo se muestra a continuación, para una mayor ilustración.

\section{Asesoria y Seguimiento}

- Proceso de acompañamiento en las acciones del proyecto educativo, promoviendo su calidad en forma permanente, superando dificultades y afirmando logros.

\section{Evaluación}

\section{- $\quad$ Proceso Participativo}

- Favorece la construcción permante del proceso

- Objetiviza y concreta la realidad de los resultados
2. Universidad de Cartagena, Facultad de Ciencias Sociales y Educación. Proyecto de Cualificación y Formación del Maestro en el Distrito de Cartagena. Formación y Construcción de Proyectos Educativos Institucionales. Cartagena, 1995.p1 3. Op. Cit.,p.5 (Ojo la nota de pié de pág \# 2 está en la pág anterior) 


\section{Taller de Campo con la Comunidad Educativa}

Proceso fundamental del proyecto que consolida la participación de todos los actores en la construcción P.E.I. a partir de:

- Elaboración de diagnosticos de las necesidades educativas de la comunidad

- Programación de acciones colectivas

- Elementos constitutivos del proyecto educativo

\section{Proceso de Desarrollo Comunitario}

- $\quad$ Espacio de trabajo en la comunidad y con la comunidad educativa

- Importancia de la participación y el reconocimiento de deberes, derechos, valores e identidad local

- Los actores son sujetos activos y responsables de los procesos que se gestionan al interior de la comunidad educativa

\section{La Participación de la Comunidad}

- $\quad$ Eje de transformación socio cultural

- Eje de articulación de los proyectos educativos institucionales

\section{Estructura y Desarrollo del Proceso}

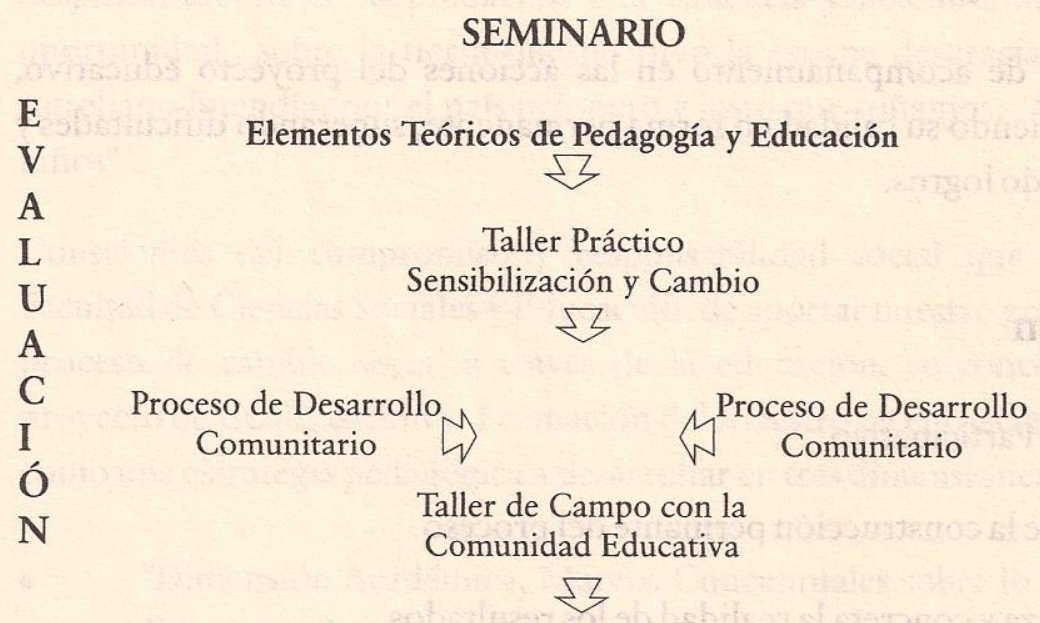

Asesoría y Monitoreo 


\section{Seminarios}

\section{Conceptos de Educación y Pedagogía}

- Encuentro con las teorias y las grandes concepciones de educación y pedagogía

- Propuesta de un proyecto educativo nacional

- Apropiación y confrontación conceptual a nivel personal en cada uno de los participantes

\section{Talleres de Sensibilización y Apropiación}

- Son espacios de integración que facilitan el proceso de aprendizaje de las temáticas conceptuales a partir del debate, la reflexión y la introspección, permitiendo la sensibilización del maestro

Igualmente los docentes participantes adquieren el compromiso de constituirse en agentes multiplicadores del proceso formativo en sus centros educativos, con proyección a la Familia y Comunidad, ampliándose el número de beneficiarios del mismo aproximadamente a unas 600 personas. Por otra parte, se comprometen al desarrollo de un trabajo escritural, fruto de la integración del Seminario con el Taller de sensibilización.

Las temáticas desarrolladas en algunos de los Seminarios fueron: Objeto de la pedagogía:
La sociedad-El trabajo-La escuela-El niño.
-
La Institución Educativa-Institución y Estado-Institución y Saber- Institución y Estrategias Teóricas.
Los sujetos pedagógicos-El Maestro El Alumno El Directivo El Educador La Pedagogía Educar Instruir y Formar.

En el proceso de desarrollo comunitario son elementos educativos focales la participación y promoción de procesos de investigación acción participación como proceso permanente de inducción del contexto cultural, escolar, familiar y comunitario. Como estrategia colectiva de intervención social, así como la relación escuela-comunidad como eje dinamizador del desarrollo social. A este proceso se vinculan alumnas en práctica de V Semestre del Programa de Trabajo Social, quienes en forma permanente están promoviendo la activa participación 
de la comunidad circundante.

El cuerpo docente estuvo constituido por un equipo básico de profesionales altamente calificados de la Universidad del Valle y la Universidad de Cartagena, quienes conjuntamente con los participantes fueron abriendo un camino conducente a un proceso de renovación educativa a partir del desarrollo y transformación personal, de su quehacer pedagógico y entorno social, producto de sus vivencias.

Desde entonces el proyecto ha continuado en forma ininterrumpida hasta nuestros días con algunas reorientaciones, producto de la experiencia y evaluación realizada; entre otras podemos mencionar: cambio en su denominación, ahora, "Procesos Pedagógicos"; variación en las temáticas de los seminarios; elaboración de un módulo por cada temática desarrollada en los seminarios mediante un proceso de construcción colectiva donde participan docentes y estudiantes en un número aproximado para cada Módulo de 20 presenciales; realización de eventos pedagógicos en los centros educativos, y elaboración de proyectos pedagógicos.

En 1997, se publicaron y entregaron a la Secretaría de Educación y Cultura Distrital, seis (6) módulos discriminados así:

$\begin{array}{ll}\text { Módulo No.1: } & \text { Pedagogía, Educación y Formación. } \\ \text { Módulo No.2: } & \text { Sociedad Civil y Democracia. } \\ \text { Módulo No.3: } & \text { Historia de Maestros, Cuerpos y Rostros. } \\ \text { Módulo No.4: } & \text { Individuo, Comunidad, Humanidad. } \\ \text { Módulo No.5: } & \text { Quehacer Pedagógico: Gestión y Organización. } \\ \text { Módulo No.6: } & \text { Formación, Aprendizaje, Didáctica y Alternativas } \\ & \text { Pedagógicas. }\end{array}$

Además, el documento titulado "Hacia la Construcción de un Modelo Pedagógico para la Formación de Maestros", con la finalidad de que fueran distribuidas en las Alcaldías Menores, Centros Educativos, Maestros Presenciales.

En 1999 a raíz de la convocatoria que la Secretaría de Educación y Cultura hiciera para la Cualificación y Formación de Maestros, la Facultad de Ciencias Sociales y Educación, presenta a la misma para estudio, la propuesta de Cualificación y Formación de Maestros en Procesos Pedagógicos con nuevas variaciones en la estructura temática de los Seminarios, por iguales razones, producto de la experiencia y evaluación realizada, éstas relacionadas con el ordenamiento y temáticas a trabajar, quedaron conformados de la siguiente manera: 
Primer Seminario: "Pedagogía, Educación y Formación".

Segundo Seminario: "Individuo Comunidad Humanidad".

Tercer Seminario: "Sociedad Civil y Democracia".

Cuarto Seminario: "Cultura y Organización Pedagógica".

Quinto Seminario: "Pedagogía, Escuela, Familia, Cultura, Comunidad".

SextoSeminario: "Formación, Aprendizaje, Didáctica y Alternativas

Pedagógicas".

En comunicación de fecha 20 de Junio de 2000 dirigida a la Rectoría de la Universidad, suscrita por la Coordinadora de Investigación y Capacitación S.E.C.P. de la Secretaría de Educación y Cultura Distrital se informa que fue emitido concepto favorable por parte del Comité Pedagógico de la antes mencionada Secretaría, anexándose copia de la Resolución 0020 del 8 de Junio de 2000, en la cual se reconoce como curso válido para el ascenso en el escalafón nacional.

A partir del año 2000, con la nueva aprobación, la Facultad de Ciencias Sociales y Educación ha venido ofreciendo a los Maestros del Distrito, hasta nuestros días la posibilidad de vincularse al proceso de Cualificación y Formación de Maestros.

En 1996, en forma simultánea al proceso de desarrollo del proyecto anterior, se amplió el ámbito de Cualificación y Formación de Maestros del Distrito de Cartagena, a docentes de instituciones educativas oficiales ubicadas dentro de la Zona Industrial de Mamonal, Área de la Refinería de Cartagena con un enfoque integral, y características específicas, mediante convenio celebrado en esa la Empresa Colombiana de Petróleos - ECOPETROL - y la Universidad de Cartagena.

Durante el desarrollo del proyecto se han trabajado entre otras las siguientes temáticas: Proyecto Educativo Institucional, Corrientes Pedagógicas, Estrategias Pedagógicas, Diseño del Plan de Estudio, Planeación Estrategia y Gestión Educativa, Contexto Escolar, Formulación de Proyectos Educativos y Sociales, Organización y Desarrollo Comunitario, Valores y Ética Personal Familiar y Social, Legislación Educativa, Construcción de Textos, Automotivación, Desarrollo Sostenible del contexto.

En 1998, el convenio se hace tripartita: Empresa Colombiana de Petróleos ECOPETROL - Alcaldía de Cartagena y Universidad de Cartagena, contemplándose en él, la Construcción Colectiva de Módulos con didácticas específicas que puedan utilizar los Maestros como textos en diferentes áreas del aprendizaje. 
4. Universidad de Cartagena. Convenio de cooperación entre la Universidad de Cartagena y la Fundación Grupo Colombo Suizo de Pedagogía Social. Cartagena 1997, P.1
Los módulos fueron elaborados por los maestros, con el acompañamiento y seguimiento de un tutor, docente de la Facultad; se conformó un Comité, con la responsabilidad de seleccionar el que a su juicio ameritara publicación por parte de la Universidad, fue así como se seleccionó y publicó el texto: "La LectoEscritura en las Ciencias Naturales"; la Universidad posteriormente, hace entrega del mismo a las partes que intervienen en el proceso de formación.

La cualificación y formación integral de maestros de la Zona Industrial de Mamonal, Área de Influencia de la Refinería, ha venido realizándose desde 1996, en forma constante, hasta el presente año (2001).

En 1997, se firma convenio de cooperación entre la Universidad de Cartagena y la Fundación Grupo Colombo Suizo de Pedagogía Especial, con el " objeto de apoyar el desarrollo de la Pedagogía Especial, a través de proyectos de capacitación, cualificación, actualización y el desarrollo de actividades conjuntas de extensión a la comunidad" ${ }^{\prime 4}$, al cual se vinculan alumnas en Prácticas del Programa de Trabajo Social.

En el antes citado convenio, igualmente se establece el compromiso de la Universidad de hacer conjuntamente con el Grupo Colombo Suizo la convocatoria para la realización del Congreso sobre Educación Especial a realizarse en 1998, así como la posibilidad de publicar previo visto bueno del Grupo Colombo-Suizo, artículos y ensayos escritos por profesionales de la Universidad de Cartagena. Con relación a los puntos mencionados, se dio cumplimiento; en lo referente a publicación, se hizo en la Revista del Grupo Colombo Suizo de dos artículos de tres docentes de la Facultad de Ciencias Sociales y Educación.

Vale la pena anotar que el convenio suscrito entre la Universidad de Cartagena y el Grupo Colombo Suizo de Pedagogía Especial, fue prorrogado a partir del $1^{\circ}$ de Abril de 1998, como aparece en constancia firmada en Marzo 31 de 1998, por la doctora Thelma del Castillo de Salazar como Rectora (e) y Pascal Afftolter, Director de la Fundación Grupo Colombo Suizo.

En 1997, al mismo tiempo en que se desarrollaban los proyectos de Cualificación y Formación de Maestros en Procesos Pedagógicos, Formación Integral en el área de influencia de ECOPETROL, y el Convenio de Cooperación entre la Universidad de Cartagena y la Fundación Grupo Colombo Suizo, a nivel Distrital, se inicia un nuevo proceso de Cualificación y Formación ahora también en el Departamento, enfocado a los "Procesos de Gestión" previo estudio y aprobación del Comité Departamental de Capacitación el que 
se desarrolló en Convenio con la Gobernación de Bolívar, en varios Municipios del Departamento de Bolívar, definidos como puntos de encuentros para tal fin, tales como: Turbaco, Magangue, Mompox, entre otras, adonde debían asistir los participantes del mismo municipio, así como las de los Municipios y Corregimientos cercanos y a donde se desplazaba el cuerpo docente responsable de su ejecución.

Para facilitar la organización y ejecución del proyecto, y garantizar su buena marcha, se nombró un coordinador general de éste, al igual que un coordinador por Municipio de encuentro, los cuales debían estar en permanente comunicación.

En el periodo del 21 de Agosto de 1998, al 19 de Febrero de 1999, se desarrolló en la Facultad de Ciencias Sociales y Educación, el Proyecto Gestión Educativa, en convenio con la Asociación de Padres de Familia de la Escuela "María Cano", para directivos y docentes de Centros Educativos del Departamento de Bolívar, "vinculados al Programa "Escuelas Amigas de los Niños, promovida por Plan Internacional, para coadyuvar en la construcción de los diferentes proyectos de formación que surjan de cada institución educativa los cuales servirán de base para el gran proyecto de Desarrollo Educativo del Departamento de Bolívar" 5 .

Las temáticas trabajadas fueron: Fundamentos Jurídicos en el Quehacer Educativo, Gestión de Centros Educativos, La Escuela como espacio del ejercicio de la Democracia, Currículo y Evaluación.

La Facultad en el presente año (2001), con el fin de responder a las demandas de Formación formuladas, ha venido ofreciendo y dando continuidad a la Cualificación y formación en "procesos de gestión, educación y administrativa".

En 1999 a raíz de convocatoria que hiciera la Secretaría de Educación y Cultura Distrital, para la Cualificación y Formación de Maestros del Distrito la Facultad presentó varios proyectos, habiendo sido aprobados como se dijera antes, el de "Procesos Pedagógicos", "Capacitación para el desarrollo integral del docente de la Zona de Influencia de ECOPETROL" y "Relaciones Interpersonales en el marco de la Administración prospectiva", este último presentado por primera vez, el cual trabajará la siguiente temática: Escuela Gestión Democracia y Desarrollo Humano, Procesos Sociales y Centros Educativos, La Administración Prospectiva en la Educación, Centros Educativos y Trabajos en Grupos, siendo con él tres los proyectos que en Cualificación y Formación de Maestros, a nivel del distrito, tiene la Facultad de Ciencias Sociales y Educación para ofrecer y desarrollar, según consta en la Resolución $N^{\circ} 0020$ de Junio 8 de 2000, de la Secretaría de Educación y Cultura Distrital.
5. Universidad de Cartagena. B or rador Convenio Interinstitucional entre la Universidad de Cartagena y la Asociación de Padres de Familia de la Escuela "María Cano". Cartagena, 1998. P1 
Desde el primer semestre del año 2001, la Facultad ha venido realizando en forma simultánea en sus Claustros, el proceso de Cualificación y Formación de Maestros a nivel Distrital y Departamental así:

Procesos Pedagógicos

Capacitación Integral del Docente en la Zona de Influencia de ECOPETROL. Relaciones Interpersonales en el Marco de la Administración Prospectiva.

Procesos de Gestión Educativa Administrativa.

Para finalizar podemos decir que en estos momentos a nivel de Facultad, se está trabajando en la elaboración de una propuesta sobre Pedagogía del Conflicto, de las Matemáticas y Lengua Castellana, a realizarse con 240 maestros de 22 Centros Educativos del área de influencia de Mamonal.

Igualmente, ha sido un camino recorrido de trabajo de seis años continuos, con muchas y valiosas experiencia de aprendizaje, y en donde la Universidad de Cartagena, a través de la Facultad de Ciencias Sociales y Educación a través de sus funciones de Docencia, Investigación y Proyección social, ha buscado contribuir al cambio social, mediante el proceso de renovación educativa.

\section{BIBLIOGRAFÍA}

GARCÍA Márquez Gabriel. La Proclama Colombia al Filo de la Oportunidad. Tercer Mundo Editores. Bogotá.1996

Universidad de Cartagena, Facultad de Ciencias Sociales y Educación. Proyecto de Cualificación y Formación del Maestro en el Distrito de Cartagena, Formación y Construcción de Proyectos educativos Institucionales. Cartagena,1995 Universidad de Cartagena, Facultad de Ciencias Sociales y Educación. Proyecto de Cualificación y Formación de Maestros. Facultad de Ciencias Sociales y Educación. 19962000.

Convenio Universidad de Cartagena, Empresa Colombiana de Petróleos ECOPETROL. Cartagena,1996-1998

Convenio Universidad de Cartagena, Fundación Grupo Colombo Suizo de Pedagogía Social. Cartagena,1997-1998 